\title{
Non-alcoholic fatty liver disease is independently associated with an increased prevalence of chronic kidney disease and retinopathy in type 1 diabetic patients
}

\author{
G. Targher • L. Bertolini • M. Chonchol • S. Rodella • \\ G. Zoppini · G. Lippi $\cdot$ L. Zenari • E. Bonora
}

Received: 1 February 2010 /Accepted: 22 February 2010/Published online: 6 April 2010

(C) Springer-Verlag 2010

\begin{abstract}
Aims/hypothesis Non-alcoholic fatty liver disease (NAFLD) is associated with an increased prevalence of chronic kidney disease (CKD) and retinopathy in patients with type 2 diabetes. Information on this issue is lacking for type 1 diabetes. We evaluated whether NAFLD is associated with increased prevalence of retinopathy and CKD in type 1 diabetic patients.

Methods All type 1 diabetic patients $(n=202)$ who regularly attended our diabetes clinic and did not have any clinical evidence of cirrhosis or other secondary causes of chronic
\end{abstract}

G. Targher $(\bowtie) \cdot G$. Zoppini $\cdot$ E. Bonora

Section of Endocrinology and Metabolism,

Department of Biomedical and Surgical Sciences,

University of Verona, Ospedale Civile Maggiore,

Piazzale Stefani 1,

37126 Verona, Italy

e-mail: giovanni.targher@univr.it

L. Bertolini $\cdot$ L. Zenari

Diabetes Unit, Sacro Cuore Hospital of Negrar (VR),

Verona, Italy

M. Chonchol

Division of Renal Diseases and Hypertension,

University of Colorado Denver Health Sciences Center,

Aurora, CO, USA

S. Rodella

Department of Radiology, Sacro Cuore Hospital of Negrar (VR),

Verona, Italy

\section{G. Lippi}

Laboratory of Clinical Chemistry,

Department of Pathology and Laboratory Medicine,

University Hospital of Parma,

Parma, Italy liver disease were studied. Main study measures were detection of NAFLD (by patient history and liver ultrasound), diabetic retinopathy (diagnosed by ophthalmoscopy) and CKD (defined as abnormal albuminuria or estimated GFR of $\left.\leq 60 \mathrm{ml} \mathrm{min}^{-1} 1.73 \mathrm{~m}^{-2}\right)$.

Results The age- and sex-adjusted prevalence of diabetic retinopathy (53.2 vs $19.8 \%$ ) and CKD (37.8 vs 9.9\%) was markedly higher in patients with NAFLD than in those without $(p<0.0001)$. In multivariate logistic regression analysis, NAFLD was associated with prevalent retinopathy (adjusted OR 3.31, 95\% CI 1.4-7.6, $p=0.005$ ) or CKD (adjusted OR $3.90,95 \%$ CI $1.5-10.1, p=0.005$ ). These associations were independent of age, sex, diabetes duration, $\mathrm{HbA}_{1 \mathrm{c}}$, medication use and presence of the metabolic syndrome.

Conclusions/interpretation Our findings suggest that ultrasound-diagnosed NAFLD is associated, independently of several confounding factors, with a higher prevalence of CKD and retinopathy in type 1 diabetic individuals.

Keywords Chronic kidney disease $\cdot$ CKD $\cdot$ Liver disease . Liver fat $\cdot$ Metabolic syndrome $\cdot$ Microvascular complications $\cdot$ NAFLD $\cdot$ Non-alcoholic fatty liver disease Retinopathy Type 1 diabetes

$\begin{array}{ll}\text { Abbreviations } \\ \text { ACR } & \text { Albumin:creatinine ratio } \\ \text { ALT } & \text { Alanine aminotransferase } \\ \text { CKD } & \text { Chronic kidney disease } \\ \text { CRP } & \text { C-reactive protein } \\ \text { CVD } & \text { Cardiovascular disease } \\ \text { MDRD } & \text { Modification of diet in renal disease } \\ \text { NAFLD } & \text { Non-alcoholic fatty liver disease } \\ \text { NASH } & \text { Non-alcoholic steatohepatitis } \\ \text { PAI-1 } & \text { Plasminogen activator inhibitor-1 }\end{array}$

Abbreviations

ACR Albumin:creatinine ratio

ALT Alanine aminotransferase

CKD Chronic kidney disease

CRP C-reactive protein

CVD Cardiovascular disease

MDRD Modification of diet in renal disease

NAFLD Non-alcoholic fatty liver disease

PAI-1 Plasminogen activator inhibitor-1 


\section{Introduction}

Non-alcoholic fatty liver disease (NAFLD) is increasingly diagnosed worldwide and considered to be the most common cause of chronic liver disease in Western countries [1-3]. NAFLD encompasses a spectrum of liver diseases ranging from simple steatosis (fatty liver) to non-alcoholic steatohepatitis (NASH) and cirrhosis [1-3].

Increased cardiovascular disease (CVD) morbidity and mortality rates are perhaps the most important clinical features associated with NAFLD, an aspect about which knowledge is rapidly evolving. Mounting evidence indicates that CVD is the leading cause of death in patients with NAFLD and that NAFLD is associated with an increased risk of incident CVD events, this association being independent of established risk factors, including components of the metabolic syndrome, in non-diabetic and type 2 diabetic individuals $[4,5]$.

A recent large study of approximately 2,000 unselected type 2 diabetic patients reported that NAFLD is independently associated with increased prevalence of diabetic retinopathy and chronic kidney disease (CKD) [6]. Since diabetic retinopathy and nephropathy represent a major problem for patients and healthcare systems $[7,8]$, the possible impact of NAFLD on the development of these chronic complications deserves particular attention, especially with regard to the implications for screening/surveillance strategies in the growing number of NAFLD patients.

To our knowledge, however, there is a gap in the knowledge of associations of NAFLD with CKD and retinopathy in patients with type 1 diabetes. As the natural history of diabetic nephropathy in type 1 diabetes is different from that in type 2 diabetes, we aimed to assess whether NAFLD as diagnosed by ultrasound, i.e. the most widely used imaging test for detecting hepatic steatosis, is associated with increased prevalence of diabetic nephropathy and retinopathy in type 1 diabetic adults.

\section{Methods}

Participants All patients diagnosed with type 1 diabetes $(n=$ 301) who regularly attended our diabetes clinic from January to December 2009 were considered eligible for the study. The following patients were excluded: (1) patients $(n=51$, $16.9 \%$ of total) for whom a liver ultrasound examination was not available; and (2) those $(n=48)$ with any clinical evidence of cirrhosis or other secondary causes of chronic liver disease (i.e. alcohol intake $>20 \mathrm{~g} /$ day, viral hepatitis, autoimmune hepatitis, haemochromatosis and use of hepato-toxic medications). The remaining $202(67.1 \%)$ type 1 diabetic patients were included in the study. There were no significant differences in age, sex, main laboratory variables and microvascular complication status between the 202 patients included in the study and those $(n=51)$ for whom liver ultrasound examination was not available (data not shown).

The local Ethics Committee approved the protocol. All participants gave their informed consent.

Clinical measurements and laboratory procedures BMI was calculated by dividing weight in kilograms by height in metres squared. Blood pressure was measured with a standard mercury manometer. Information on daily alcohol consumption, smoking status and current use of medications was obtained from all participants by questionnaire [9].

Venous blood was drawn in the morning after an overnight fast. Serum liver enzymes, creatinine (measured using a Jaffé rate-blanked and compensated assay) and other biochemical blood measurements were determined by standard laboratory procedures (DAX 96; Bayer Diagnostics, Milan, Italy). Normal ranges for serum aminotransferase levels in our laboratory were 10-40 U/1 for men and women. LDLcholesterol was calculated by Friedewald's equation, except when triacylglycerol exceeded $4.55 \mathrm{mmol} / \mathrm{l} . \mathrm{HbA}_{1 \mathrm{c}}$ was measured using a high-performance liquid chromatography analyser (HA-8140; Menarini Diagnostics, Florence, Italy); the upper limit of normal for the laboratory was $5.9 \%$. GFR was estimated from the four-variable modification of diet in renal disease (MDRD) equation [10]. Urinary AER was measured from an early morning urine sample as the albumin: creatinine ratio (ACR) by an immuno-nephelometric method; microalbuminuria and macroalbuminuria were defined as ACR $>2.5$ and $>30 \mathrm{mg} / \mathrm{mmol}$ for men and ACR $>3.5$ and $>30 \mathrm{mg} / \mathrm{mmol}$ for women, respectively [11]. For this study, CKD was defined as abnormal albuminuria and/or estimated GFR $\leq 60 \mathrm{ml} \mathrm{min}{ }^{-1} 1.73 \mathrm{~m}^{-2}[10,11]$. Both of these measures were confirmed in all participants in a least two consecutive occasions (within 3 to 6 months after the initial examination).

Metabolic syndrome was diagnosed by a modified Adult Treatment Panel (ATP) III definition because waist circumference was available only in few participants. In accordance with this modified ATP III definition [12, 13], a person with type 1 diabetes was classified as having the metabolic syndrome if he/she had at least two of the following four components: (1) BMI $>28 \mathrm{~kg} / \mathrm{m}^{2}$ in men or $>27 \mathrm{~kg} / \mathrm{m}^{2}$ in women; (2) triacylglycerol $\geq 1.7 \mathrm{mmol} / 1$; (3) HDLcholesterol $<1.0 \mathrm{mmol} / 1$ in men and $<1.29 \mathrm{mmol} / 1$ in women or receiving lipid-lowering medications; and (4) blood pressure $\geq 130 / 85 \mathrm{mmHg}$ or receiving antihypertensive medications.

A single ophthalmologist, who was blinded to clinical patients' characteristics, diagnosed retinopathy by fundoscopy after pupillary dilation, according to a clinical disease severity scale $[6,14]$. Retinopathy was classified into four categories as follows: absent, non-proliferative, 
proliferative or laser-treated retinopathy [6]. Proliferative retinopathy was confirmed by fundus fluorescein angiography in all participants.

Hepatic ultrasonography scanning was performed in all patients by an experienced radiologist (S. Rodella), who was blinded to participants' details. Hepatic steatosis was diagnosed on the basis of characteristic sonographic features. A $3.5 \mathrm{MHz}$ transducer was used to obtain the following images: sagittal view of the right lobe of the liver and right kidney; transverse view of the left lateral segment of the liver and spleen; transverse view of the liver and pancreas; and any focal areas of altered echotexture. On ultrasonography, the steatotic liver of mild, moderate or severe degree is (finely and diffusely) hyperechoic compared with the cortex of the right kidney (so-called 'bright liver'). In addition, in moderate and severe steatosis, there is also a slightly or markedly posterior attenuation of the ultrasound beam and reduced visualisation of intra-hepatic vessel borders and diaphragm [15-17]. Several studies have assessed the sensitivity and specificity of ultrasonography for detecting hepatic steatosis. In these, the sensitivity ranged from $60 \%$ to $94 \%$ and the specificity from $84 \%$ to $95 \%$, respectively [17]. It is known that liver ultrasonography has good sensitivity and specificity in detection of moderate and severe steatosis, but its sensitivity is reduced in morbidly obese individuals or when hepatic fat infiltration upon liver biopsy is $<33 \%$ [15-17]. Moreover, ultrasonography is unable to provide a precise determination of intrahepatic fat content and cannot distinguish between steatosis alone and NASH, or stage the degree of liver fibrosis [1517]. Semi-quantitative sonographic scoring for the degree of hepatic steatosis was not available in this study. Grading of hepatic fat content using ultrasound has been used in previous studies but remains somewhat subjective [17]. As previously reported $[6,9]$, intra-observer variability for the ultrasound diagnosis of hepatic steatosis was within $3 \%$.

Statistical analysis Data are presented as means \pm SD or frequencies. Skewed variables (triacylglycerol and liver enzymes) were logarithmically transformed to improve normality prior to analysis. The unpaired $t$ test and the $\chi^{2}$ test with Yates's correction for continuity (for categorical variables) were used to compare the baseline clinical characteristics of participants stratified by the presence or absence of ultrasound-diagnosed NAFLD. Logistic regression analysis was used to assess the independent association of NAFLD with CKD, retinopathy or both complications (considered as composite endpoint) after adjustment for potential confounders. Four forced entry multivariate logistic regression models were performed. The first regression model was adjusted for age and sex (model 1); the second regression model was adjusted for age, sex, diabetes duration and $\mathrm{HbA}_{1 \mathrm{c}}$ (model 2); the third regression model was further adjusted for the presence/absence of the metabolic syndrome (model 3); and finally, in the fourth regression model, the presence of the metabolic syndrome was replaced by BMI and other individual components of metabolic syndrome, included as continuous measures, and by the use of anti-hypertensive or lipid-lowering drugs (model 4). The covariates included in multivariate regression models were chosen as potential confounders based on their biological plausibility or statistical association with retinopathy and CKD in univariate analyses. Results are presented as ORs with $95 \%$ CIs. Values of $p<0.05$ were considered statistically significant.

\section{Results}

Overall, the 202 participants of the study had a mean age of 43.2 years, mean BMI of $24.8 \mathrm{~kg} / \mathrm{m}^{2}$, mean $\mathrm{HbA}_{1 \mathrm{c}}$ of $8.2 \%$ and mean duration of disease of 18.5 years, respectively. Of these participants, 91 (45.1\%) patients had negative liver ultrasound tests as well as normal serum liver enzymes in the absence of excessive alcohol consumption, viral hepatitis or use of hepato-toxic medications, whereas the remaining $111(54.9 \%)$ patients met the criteria for diagnosing NAFLD.

The clinical and biochemical characteristics of patients stratified by their NAFLD status are shown in Table 1 . Patients with NAFLD were older, more likely to be obese and male, and had longer duration of diabetes and higher frequency of the metabolic syndrome and abnormal albuminuria than those without NAFLD. They also had lower estimated GFR and higher $\mathrm{HbA}_{1 \mathrm{c}}$, triacylglycerol and liver enzymes, although the vast majority of NAFLD patients, i.e. $80.2 \%$, had serum alanine aminotransferase (ALT) concentrations within the reference range. Smoking history and HDL-cholesterol were not significantly different between the groups. The proportion using anti-hypertensive (55.0 vs $14.3 \%$ ) or lipid-lowering ( 38.0 vs $6.6 \%$ ) medications was higher $(p<0.0001)$ in NAFLD patients.

Overall, 77 (38.1\%) of 202 patients had some degree of diabetic retinopathy (64 non-proliferative retinopathy and 13 either proliferative or laser-treated retinopathy) and 51 (25.2\%) had CKD defined as abnormal albuminuria or estimated GFR $\leq 60 \mathrm{ml} \mathrm{min}^{-1} 1.73 \mathrm{~m}^{-2}$ (40 with micro- or macro-albuminuria with estimated GFR $>60 \mathrm{ml} \mathrm{min}^{-1}$ $1.73 \mathrm{~m}^{-2}, 11$ with an estimated GFR $\leq 60 \mathrm{ml} \mathrm{min}{ }^{-1} 1.73 \mathrm{~m}^{-2}$ irrespective of albuminuria). A total of 94 (46.5\%) patients had retinopathy and/or CKD (composite endpoint).

Notably, as shown in Fig. 1, patients with NAFLD had a remarkably higher $(p<0.0001)$ age- and sex-adjusted prevalence of diabetic retinopathy, CKD or both than their counterparts without NAFLD. 
Table 1 Baseline characteristics of the study participants stratified by NAFLD status
Data are expressed as means \pm SD or proportions $(\%)$

AST, aspartate aminotransferase; GGT, gamma-glutamyltransferase

\begin{tabular}{|c|c|c|c|}
\hline Characteristic & Without fatty liver & With NAFLD & $p$ value \\
\hline$n$ & 91 & 111 & \\
\hline Sex $(\%$ men $)$ & 40 & 63 & 0.001 \\
\hline Age (years) & $37 \pm 12$ & $47 \pm 12$ & $<0.0001$ \\
\hline BMI $\left(\mathrm{kg} / \mathrm{m}^{2}\right)$ & $22.5 \pm 3$ & $26.6 \pm 5$ & $<0.0001$ \\
\hline Current smokers $(\%)$ & 18.7 & 19.8 & 0.610 \\
\hline Systolic BP (mmHg) & $118 \pm 15$ & $129 \pm 16$ & $<0.0001$ \\
\hline Diastolic BP (mmHg) & $71 \pm 8$ & $76 \pm 7$ & $<0.0001$ \\
\hline Diabetes duration (years) & $14 \pm 11$ & $22 \pm 12$ & $<0.0001$ \\
\hline $\mathrm{HbA}_{1 \mathrm{c}}(\%)$ & $8.0 \pm 1.1$ & $8.4 \pm 1.3$ & 0.046 \\
\hline LDL-cholesterol (mmol/l) & $2.72 \pm 0.7$ & $2.92 \pm 0.7$ & 0.042 \\
\hline HDL-cholesterol (mmol/l) & $1.52 \pm 0.4$ & $1.45 \pm 0.4$ & 0.163 \\
\hline Triacylglycerol (mmol/l) & $0.77 \pm 0.3$ & $1.53 \pm 1.4$ & $<0.0001$ \\
\hline Estimated GFR (ml $\left.\min ^{-1} 1.73 \mathrm{~m}^{-2}\right)$ & $107 \pm 28$ & $98 \pm 25$ & 0.014 \\
\hline Micro- or macro-albuminuria (\%) & 4.4 & 36.4 & $<0.0001$ \\
\hline Metabolic syndrome (\%) & 11 & 62.2 & $<0.0001$ \\
\hline AST (U/1) & $20 \pm 6$ & $25 \pm 11$ & $<0.0001$ \\
\hline ALT (U/l) & $20 \pm 8$ & $29 \pm 17$ & $<0.0001$ \\
\hline GGT (U/l) & $17 \pm 9$ & $29 \pm 20$ & $<0.0001$ \\
\hline Elevated ALT (>40 U/1) (\%) & 4.4 & 19.8 & 0.001 \\
\hline
\end{tabular}

In unadjusted logistic regression analysis (Table 2), NAFLD was significantly associated with retinopathy and/ or CKD. Age, duration of diabetes, systolic blood pressure, $\mathrm{BMI}, \mathrm{HbA}_{1 \mathrm{c}}$ and the presence of the metabolic syndrome were also significantly associated $(p<0.01-0.001)$ with either retinopathy or $\mathrm{CKD}$ in unadjusted regression analyses (data not shown).

Table 3 shows the association of NAFLD with either retinopathy or CKD in subgroup analyses. Interestingly, NAFLD was significantly associated with retinopathy and/ or CKD even when the study population was stratified according to sex distribution and to the median values of age, diabetes duration, $\mathrm{BMI}$ or $\mathrm{HbA}_{1 \mathrm{c}}$.

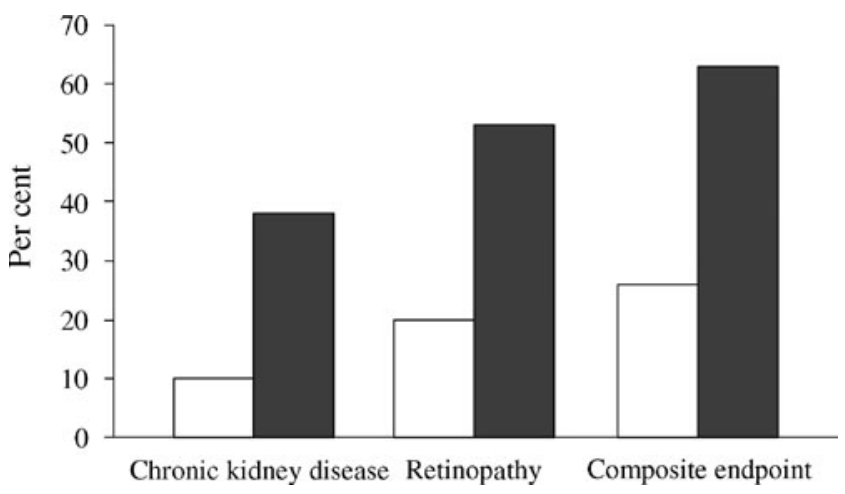

Fig. 1 Age- and sex-adjusted prevalence of chronic kidney disease, retinopathy and/or both (composite endpoint) in type 1 diabetic adults with (black columns) and without (white columns) ultrasounddiagnosed NAFLD. $p<0.0001$ for differences between those with vs those without NAFLD
Table 2 Associations of ultrasound-diagnosed NAFLD with diabetic retinopathy and $\mathrm{CKD}$ in type 1 diabetic individuals

\begin{tabular}{llc}
\hline Variable & OR $(95 \% \mathrm{CI})$ & $p$ value \\
\hline CKD & & \\
Unadjusted & $5.51(2.5-12.5)$ & $<0.0001$ \\
Adjusted model 1 & $4.40(1.9-10.3)$ & $<0.001$ \\
Adjusted model 2 & $4.16(1.7-10.0)$ & 0.001 \\
Adjusted model 3 & $3.90(1.5-10.1)$ & 0.005 \\
Adjusted model 4 & $3.29(1.2-9.1)$ & $<0.01$ \\
Diabetic retinopathy & & $<0.0001$ \\
Unadjusted & $4.60(2.4-8.7)$ & $<0.001$ \\
Adjusted model 1 & $3.67(1.8-7.4)$ & 0.001 \\
Adjusted model 2 & $3.62(1.7-7.9)$ & 0.005 \\
Adjusted model 3 & $3.31(1.4-7.6)$ & $<0.01$ \\
Adjusted model 4 & $3.19(1.3-7.5)$ & $<0.0001$ \\
CKD and/or retinopathy & & $<0.001$ \\
Unadjusted & $4.77(2.6-8.7)$ & $<0.001$ \\
Adjusted model 1 & $3.62(1.8-6.9)$ & $<0.001$ \\
Adjusted model 2 & $3.53(1.7-7.3)$ & $<0.01$ \\
Adjusted model 3 & $3.71(1.7-8.2)$ & \\
Adjusted model 4 & $3.25(1.4-7.1)$ & \\
\hline
\end{tabular}

Data are expressed as $\mathrm{OR} \pm 95 \% \mathrm{CI}$ as assessed by univariate (unadjusted) or multivariate logistic regression analysis

Models adjusted as follows: model 1: age and sex; model 2: age, sex, diabetes duration and $\mathrm{HbA}_{1 \mathrm{c}}$; model 3: as model 2, plus further adjustment for presence of the metabolic syndrome (as categorical variable); model 4: as model 2 plus BMI, systolic BP, triacylglycerol and medication use (i.e. antihypertensive or lipid-lowering drugs) 
Table 3 Associations of NAFLD with CKD or retinopathy in type 1 diabetic individuals stratified according to sex distribution or to the median values of age, diabetes duration, $\mathrm{BMI}$ and $\mathrm{HbA}_{1 \mathrm{c}}$

\begin{tabular}{|c|c|c|}
\hline Variable & OR $(95 \% \mathrm{CI})$ & $p$ value \\
\hline \multicolumn{3}{|l|}{ CKD } \\
\hline Men $(n=106)$ & $10.6(2.4-47.0)$ & $<0.005$ \\
\hline Women $(n=96)$ & $4.01(1.4-11.0)$ & $<0.01$ \\
\hline Age $<43$ years $(n=105)$ & $11.0(2.6-41.0)$ & $<0.001$ \\
\hline Age $\geq 43$ years $(n=97)$ & $2.01(1.3-5.52)$ & $<0.01$ \\
\hline Diabetes duration $<15$ years $(n=100)$ & $8.80(1.8-41.7)$ & $<0.005$ \\
\hline Diabetes duration $\geq 15$ years $(n=102)$ & $3.91(1.5-10.0)$ & $<0.005$ \\
\hline $\mathrm{BMI}<24 \mathrm{~kg} / \mathrm{m}^{2}(n=97)$ & $4.51(1.6-13.1)$ & $<0.005$ \\
\hline $\mathrm{BMI} \geq 24 \mathrm{~kg} / \mathrm{m}^{2}(n=105)$ & $9.10(1.8-40.0)$ & $<0.005$ \\
\hline $\mathrm{HbA}_{1 \mathrm{c}}<8 \%(n=98)$ & $5.78(2.0-16.0)$ & $<0.001$ \\
\hline $\mathrm{HbA}_{1 \mathrm{c}} \geq 8 \%(n=104)$ & $6.30(1.7-22.7)$ & $<0.005$ \\
\hline \multicolumn{3}{|l|}{ Diabetic retinopathy } \\
\hline Men $(n=106)$ & $3.50(1.4-8.7)$ & $<0.01$ \\
\hline Women $(n=96)$ & $6.35(2.5-16.0)$ & $<0.0001$ \\
\hline Age $<43$ years $(n=105)$ & $2.11(1.01-4.9)$ & $<0.05$ \\
\hline Age $\geq 43$ years $(n=97)$ & $10.7(2.9-39)$ & $<0.001$ \\
\hline Diabetes duration $<15$ years $(n=100)$ & $8.70(1.8-41.0)$ & $<0.005$ \\
\hline Diabetes duration $\geq 15$ years $(n=102)$ & $3.81(1.6-8.9)$ & $<0.01$ \\
\hline $\mathrm{BMI}<24 \mathrm{~kg} / \mathrm{m}^{2}(n=97)$ & $8.50(3.2-22.6)$ & $<0.0001$ \\
\hline $\mathrm{BMI} \geq 24 \mathrm{~kg} / \mathrm{m}^{2}(n=105)$ & $3.22(1.2-8.2)$ & $<0.01$ \\
\hline $\mathrm{HbA}_{1 \mathrm{c}}<8 \%(n=98)$ & $8.20(3.0-22.0)$ & 0.0001 \\
\hline $\mathrm{HbA}_{1 \mathrm{c}} \geq 8 \%(n=104)$ & $5.35(1.2-6.34)$ & $<0.01$ \\
\hline
\end{tabular}

In multivariate logistic regression analysis (Table 2), the significant relationships of NAFLD with retinopathy, CKD or both were only slightly weakened after adjustment for age, sex, diabetes duration and $\mathrm{HbA}_{1 \mathrm{c}}$ (model 1 and 2). Additional adjustment for the presence/absence of the metabolic syndrome did not substantially change the results (model 3 ).
Almost identical results were also obtained when the metabolic syndrome (included as categorical variable) was replaced by BMI and other individual components of metabolic syndrome as continuous measures, and by the use of anti-hypertensive or lipid-lowering medications (model 4).

As specifically shown in Table 4, this latter multivariate regression model revealed that, together with NAFLD, longer duration of diabetes and the presence of hypertension (as reflected by higher systolic blood pressure values and by the current use of anti-hypertensive medications) were also independently associated with CKD and/or retinopathy.

\section{Discussion}

There is a pressing and unmet need to determine whether NAFLD is associated with a higher frequency of diabetic retinopathy and CKD in people with type 1 diabetes. It has only recently been recognised that NAFLD represents an important burden of disease for type 2 diabetic patients [4, $6,9,18,19]$, but the magnitude of the problem of NAFLD in type 1 diabetes is presently poorly recognised.

To our knowledge, this is the first cross-sectional study with the specific aim of assessing the association of NAFLD with CKD or retinopathy in type 1 diabetic individuals.

Our major finding was that NAFLD, as diagnosed by patient history and liver ultrasound, is strongly associated with an increased prevalence of diabetic retinopathy and nephropathy in unselected type 1 diabetic adults. Notably, this association appears to be independent of age, sex, diabetes duration, $\mathrm{HbA}_{1 \mathrm{c}}$, medication use, $\mathrm{BMI}$ and other components of the metabolic syndrome. Interestingly, our results also confirmed a high prevalence of the metabolic

Table 4 Multivariate logistic regression analyses using independent predictors of CKD and/or diabetic retinopathy in type 1 diabetic individuals

\begin{tabular}{|c|c|c|c|c|c|c|}
\hline \multirow[t]{2}{*}{ Independent variable } & \multicolumn{2}{|l|}{ CKD } & \multicolumn{2}{|l|}{ Retinopathy } & \multicolumn{2}{|c|}{ Composite endpoint } \\
\hline & OR $(95 \% \mathrm{CI})$ & $p$ value & OR $(95 \% \mathrm{CI})$ & $p$ value & OR $(95 \% \mathrm{CI})$ & $p$ value \\
\hline Age (years) & $0.98(0.94-1.03)$ & 0.39 & $0.98(0.94-1.02)$ & 0.39 & $1.00(0.97-1.04)$ & 0.89 \\
\hline Sex (male vs female) & $0.86(0.41-1.92)$ & 0.70 & $1.10(0.53-2.32)$ & 0.80 & $1.00(0.51-2.10)$ & 0.90 \\
\hline Diabetes duration (years) & $1.06(1.02-1.11)$ & $<0.005$ & $1.10(1.06-1.15)$ & $<0.001$ & $1.09(1.05-1.14)$ & $<0.001$ \\
\hline $\mathrm{HbA}_{1 \mathrm{c}}(\%)$ & $0.94(0.70-1.28)$ & 0.65 & $1.02(0.76-1.36)$ & 0.92 & $0.98(0.74-1.31)$ & 0.86 \\
\hline BMI $\left(\mathrm{kg} / \mathrm{m}^{2}\right)$ & $0.98(0.90-1.05)$ & 0.30 & $1.01(0.92-1.11)$ & 0.80 & $1.00(0.90-1.11)$ & 0.94 \\
\hline Systolic BP (mmHg) & $1.05(1.02-1.10)$ & $<0.01$ & $0.99(0.96-1.02)$ & 0.51 & $0.99(0.97-1.03)$ & 0.61 \\
\hline Triacylglycerol (mmol/1) & $1.01(1.00-1.02)$ & 0.052 & $1.01(0.99-1.00)$ & 0.70 & $1.00(0.99-1.02)$ & 0.40 \\
\hline Antihypertensive drug use (yes/no) & $2.01(0.90-4.8)$ & 0.08 & $3.40(1.51-7.82)$ & $<0.005$ & $2.80(1.21-6.60)$ & $<0.01$ \\
\hline Lipid-lowering drug use (yes/no) & $0.73(0.30-1.74)$ & 0.40 & $0.62(0.23-1.50)$ & 0.60 & $0.49(0.20-1.10)$ & 0.40 \\
\hline NAFLD (yes/no) & $3.29(1.2-9.1)$ & $<0.01$ & $3.19(1.3-7.5)$ & $<0.01$ & $3.25(1.4-7.1)$ & $<0.01$ \\
\hline
\end{tabular}

Values are OR $(95 \% \mathrm{CI})$, participants $n=202$ 
syndrome in type 1 diabetic patients [20-22], which is closely associated with NAFLD.

Another major finding of our study was that the frequency of ultrasound-diagnosed NAFLD is high (i.e. $\sim 50 \%$ ) in type 1 diabetic adults and that the vast majority of NAFLD patients $(80.2 \%)$ have serum ALT concentrations within the reference range. Even when more stringent criteria were used (i.e. serum ALT $>30 \mathrm{U} / 1$ in men and $>19$ U/1 in women) [23], nearly half of our NAFLD patients (48\%) had normal ALT levels. This finding provides further evidence that serum ALT and other liver enzymes appear to be insensitive markers for detection of NAFLD. Indeed, the full histological spectrum of NAFLD may be present among patients with 'normal' liver enzymes, which therefore cannot be reliably used to exclude the presence of more advanced stages of NAFLD [24]. Our finding further supports the notion that the 'normal' reference values for serum liver enzymes currently used to exclude NAFLD need to be challenged and revised $[1,2,25]$.

While there is now growing evidence to suggest that NAFLD, especially in its necro-inflammatory form (NASH), is closely linked to an increased risk of incident CVD events in non-diabetic and type 2 diabetic individuals $[4,5]$, the available information on the association of NAFLD with CKD or retinopathy is quantitatively limited.

A recent large study of 2,103 unselected type 2 diabetic individuals reported that, independently of several established risk factors, patients with ultrasound-diagnosed NAFLD had a remarkably higher prevalence of diabetic retinopathy and nephropathy than those without NAFLD [6]. In the same cohort of type 2 diabetic patients, who were followed for a mean period of 6.5 years, the presence of ultrasound-diagnosed NAFLD was found to be independently associated with an increased incidence of CKD [26]. Other large population-based studies using elevated serum liver enzymes, as surrogate markers of NAFLD, have also shown NAFLD to be independently associated with increased prevalence and incidence of CKD [27-30].

Given the cross-sectional design of this study, we are unable to draw conclusions about causality of the relationship between NAFLD and diabetic retinopathy or nephropathy. A plausible explanation for our findings is that the higher frequency of diabetic retinopathy and CKD among individuals with NAFLD simply reflects the coexistence of underlying known risk factors, such as age, diabetes duration, $\mathrm{HbA}_{1 \mathrm{c}}, \mathrm{BMI}$ and other components of the metabolic syndrome. However, because in our study NAFLD was associated with diabetic retinopathy and CKD independently of the above-mentioned risk factors, it is possible that NAFLD itself could, at least in part, contribute to the development of CKD and retinopathy, although the possibility of reverse causality (i.e. CKD or retinopathy causing the development of NAFLD) cannot be definitely excluded from our study. However, the evidence from recent large prospective studies indicates that NAFLD is an independent long-term predictor of incident CKD in non-diabetic and type 2 diabetic individuals [26, 29, 30].

The biological mechanisms by which NAFLD could contribute to the development or progression of diabetic retinopathy and CKD are poorly known.

As recently reviewed in detail $[31,32]$, several casecontrol studies using liver biopsies to diagnose NAFLD have consistently demonstrated that circulating levels of several inflammatory markers (e.g. C-reactive protein [CRP], IL-6, TNF-alpha, TGF-beta 1) and procoagulant factors (e.g. plasminogen activator inhibitor-1 [PAI-1], factor VII, fibrinogen) are highest in patients with NASH, intermediate in those with simple steatosis and lowest in non-steatotic healthy controls, these findings being independent of BMI and other established risk factors. Notably, some of these studies have shown a strong graded relationship between intra-hepatic mRNA expression of IL6 (also known as ILO), CRP or PAI-1 (also known as SERPINE1) and the severity of NAFLD histology [33-35]. These observations suggest that NAFLD is not only a marker of renal and retinal complications in type 1 diabetes, but also may be involved in their pathogenesis, possibly through the systemic release of pathogenic mediators from the steatotic/inflamed liver, including elevated advanced glycated end-products, increased reactive oxygen species, and elevated CRP, TNF-alpha, TGFbeta 1 and other proinflammatory cytokines [31, 32].

The potential implications of our findings for patient care are that in people with type 1 diabetes the casual detection of NAFLD during ultrasound examination should alert clinicians to the possible coexistence of chronic (micro)vascular complications warranting evaluation and treatment with the risk of advancing liver disease.

The present study has some important limitations. First, the cross-sectional study design precludes the establishment of causal or temporal relationships between NAFLD, CKD and retinopathy. Second, we used an estimated GFR (i.e. the four-variable MDRD study equation) instead of a directly measured GFR to define CKD. It is known that current GFR estimates have greater inaccuracy in populations without known CKD than in those with the disease $[10,11]$. Nonetheless, current GFR estimates facilitate the detection, evaluation and management of $\mathrm{CKD}$, and many organisations recommend the MDRD study equation for estimation of renal function in epidemiological studies and in clinical practice $[10,11]$. Third, the diagnosis of nonproliferative retinopathy was based only on fundoscopy, so it is possible that subtle retinopathy changes may have been missed. However, the possibility that subtle retinopathy changes might have gone partly unnoticed would have weakened, rather than strengthened our findings. Finally, the diagnosis of NAFLD was based on ultrasound imaging 
and the exclusion of other known aetiological factors of chronic liver disease, but was not confirmed by liver biopsy, which is not easily applied in epidemiological studies. It is known that none of the radiological features can distinguish between NASH and other forms of NAFLD, and that only liver biopsy can assess the severity of damage and the prognosis [1,2, 15-17]. However, liver ultrasound is the most widely used non-invasive technique to detect fatty infiltration of the liver in clinical practice. It has a good sensitivity and specificity in detecting moderate and severe hepatic steatosis, but this sensitivity is reduced in morbidly obese individuals or when hepatic fat infiltration upon liver biopsy is less than 33\% [15-17]. Thus, although some non-differential misclassification of NAFLD (fatty liver) on the basis of ultrasonography is likely (some of the diabetic control patients could have underlying NAFLD despite normal serum liver enzymes and negative ultrasonography examination), this limitation would serve to attenuate the magnitude of our effect measures toward null; thus, our results can probably be considered to be conservative estimates of the relationship between NAFLD, CKD and retinopathy

In conclusion, our results suggest that ultrasounddiagnosed NAFLD is associated with a remarkably higher prevalence of diabetic retinopathy and nephropathy, independently of established risk factors. These cross-sectional findings, although not definitive, are sufficiently provocative and hypothesis-generating to warrant further study. Further studies using more accurate non-invasive imaging techniques for measuring fatty infiltration of the liver are required to confirm the reproducibility of these results. Moreover, prospective studies are also needed to determine whether NAFLD contributes to the development of CKD and retinopathy in type 1 and also type 2 diabetes.

Duality of interest The authors declare that there is no duality of interest associated with this manuscript.

\section{References}

1. Adams LA, Lindor KD (2007) Nonalcoholic fatty liver disease. Ann Epidemiol 17:863-869

2. de Alwis NMW, Day CP (2008) Nonalcoholic fatty liver disease: the mist gradually clears. J Hepatol 48(Suppl 1):S104-S112

3. Kotronen A, Yki-Järvinen H (2008) Fatty liver: a novel component of the metabolic syndrome. Arterioscler Thromb Vasc Biol 28:27-38

4. Targher G, Marra F, Marchesini (2008) Increased risk of cardiovascular disease in non-alcoholic fatty liver disease: causal effect or epiphenomenon? Diabetologia 51:1947-1953

5. Misra VL, Khashab M, Chalasani N (2009) Nonalcoholic fatty liver disease and cardiovascular risk. Curr Gastroenterol Rep 11:50-55

6. Targher G, Bertolini L, Rodella S et al (2008) Non-alcoholic fatty liver disease is independently associated with an increased prevalence of chronic kidney disease and proliferative/laser-treated retinopathy in type 2 diabetic patients. Diabetologia 51:444-450

7. Mohamed Q, Gillies M, Wong TY (2007) Management of diabetic retinopathy: a systematic review. JAMA 298:902-916

8. Rossing P (2006) Prediction, progression and prevention of diabetic nephropathy. The Minkowski Lecture 2005. Diabetologia 49:11-19

9. Targher G, Bertolini L, Padovani R et al (2007) Prevalence of nonalcoholic fatty liver disease and its association with cardiovascular disease among type 2 diabetic patients. Diabetes Care 30:1212-1218

10. Stevens LA, Coresh J, Greene T, Levey AS (2006) Assessing kidney function-measured and estimated glomerular filtration rate. N Engl J Med 354:2473-2483

11. American Diabetes Association (2010) Standards of medical care in diabetes-2010. Diabetes Care 33(Suppl 1):S11-S61

12. Sattar N, Gaw A, Scherbakova O et al (2003) Metabolic syndrome with and without $\mathrm{C}$-reactive protein as a predictor of coronary heart disease and diabetes in the West of Scotland Coronary Prevention Study. Circulation 108:414-419

13. Girman CJ, Rhodes T, Mercuri M, 4S Group and the AFCAPS/ TexCAPS Research Group et al (2004) The metabolic syndrome and risk of major coronary events in the Scandinavian Simvastatin Survival Study (4S) and the Air Force/Texas Coronary Atherosclerosis Prevention Study (AFCAPS/TexCAPS). Am J Cardiol 93:136-141

14. Wilkinson CP, Ferris FL, Klein RE, Global Diabetic Retinopathy Project Group et al (2003) Proposed international clinical diabetic retinopathy and diabetic macular edema disease severity scales. Ophthalmology 110:1677-1682

15. Joseph AE, Saverymuttu SH, al-Sam S, Cook MG, Maxwell JD (1991) Comparison of liver histology with ultrasonography in assessing diffuse parenchymal liver disease. Clin Radiol 43:26-31

16. Saadeh S, Younossi ZM, Remer EM et al (2002) The utility of radiological imaging in nonalcoholic fatty liver disease. Gastroenterology 123:745-750

17. Mehta SR, Thomas EL, Bell JD, Johnston DG, Taylor-Robinson SD (2008) Non-invasive means of measuring hepatic fat content. World J Gastroenterol 14:3476-3483

18. Targher G, Bertolini L, Poli F et al (2005) Nonalcoholic fatty liver disease and risk of future cardiovascular events among type 2 diabetic patients. Diabetes 54:3541-3546

19. Targher G, Bertolini L, Rodella S et al (2007) Nonalcoholic fatty liver disease is independently associated with an increased incidence of cardiovascular events in type 2 diabetic patients. Diabetes Care 30:2119-2121

20. Thorn LM, Forsblom C, Fagerudd J, FinnDiane Study Group et al (2005) Metabolic syndrome in type 1 diabetes. Association with diabetic nephropathy and glycemic control (the FinnDiane study). Diabetes Care 28:2019-2024

21. Pambianco G, Costacou T, Orchard TJ (2007) The prediction of major outcomes of type 1 diabetes: a 12-year prospective evaluation of three separate definitions of the metabolic syndrome and their components and estimated glucose disposal rate. The Pittsburgh Epidemiology of Diabetes Complications Study Experience. Diabetes Care 30:1248-1254

22. Kilpatrick ES, Rigby AS, Atkin SL (2007) Insulin resistance, the metabolic syndrome, and complication risk in type 1 diabetes: "double diabetes" in the Diabetes Control and Complications Trial. Diabetes Care 30:707-712

23. Prati D, Taioli E, Zanella A et al (2002) Updated definitions of healthy ranges for serum alanine aminotransferase levels. Ann Intern Med 137:1-10

24. Mofrad P, Contos MJ, Haque M et al (2003) Clinical and histological spectrum of nonalcoholic fatty liver disease associated with normal ALT values. Hepatology 37:1286-1292

25. Ratziu V, Imbert-Bismut F, Messous D, Poynard T (2004) The elusiveness of "normal" ALT in fatty liver. Hepatology 39:1172 
26. Targher G, Chonchol M, Bertolini L et al (2008) Increased risk of CKD among type 2 diabetics with nonalcoholic fatty liver disease. J Am Soc Nephrol 19:1564-1570

27. Targher G, Kendrick J, Smits G, Chonchol M (2010) Relationship between elevated serum gamma-glutamyltransferase concentrations and chronic kidney disease in the United States population. Findings from the National Health and Nutrition Examination Survey 2001-2006. Nutr Metab Cardiovasc Dis. doi:10.1016/j. numecd.2009.05.012

28. Targher G, Bosworth C, Kendrick J, Smits G, Lippi G, Chonchol M (2009) Relationship of serum bilirubin concentrations to kidney function and albuminuria in the United States adult population. Findings from the National Health and Nutrition Examination Survey 2001-2006. Clin Chem Lab Med 47:1055-1062

29. Ryu S, Chang Y, Kim DI, Kim WS, Suh BS (2007) Gammaglutamyltransferase as a predictor of chronic kidney disease in nonhypertensive and nondiabetic Korean men. Clin Chem 53:71-77

30. Lee DH, Jacobs DR, Gross M, Steffes M (2005) Serum gammaglutamyltransferase was differently associated with microalbuminuria by status of hypertension and diabetes: the Coronary Artery
Risk development in Young Adults (CARDIA) study. Clin Chem 51:1185-1191

31. Targher G, Chonchol M, Miele L, Zoppini G, Pichiri I, Muggeo M (2009) Nonalcoholic fatty liver disease as a contributor to hypercoagulation and thrombophilia in the metabolic syndrome. Semin Thromb Hemost 35:277-287

32. Edens MA, Kuipers F, Stolk RP (2009) Non-alcoholic fatty liver disease is associated with cardiovascular disease risk markers. Obes Rev 10:412-419

33. Yoneda M, Mawatari H, Fujita K et al (2007) High-sensitivity Creactive protein is an independent clinical feature of nonalcoholic steatohepatitis (NASH) and also of the severity of fibrosis in NASH. J Gastroenterol 42:573-582

34. Thuy S, Ladurner R, Volynets V et al (2008) Nonalcoholic fatty liver disease in humans is associated with increased plasma endotoxin and plasminogen activator inhibitor 1 concentrations and with fructose intake. J Nutr 138:1452-1455

35. Wieckowska A, Papouchado BG, Li Z, Lopez R, Zein NN, Feldstein AE (2008) Increased hepatic and circulating interleukin6 levels in human nonalcoholic steatohepatitis. Am J Gastroenterol 103:1372-1379 